\title{
FERMENTATION OF MULTIGRAIN DOUGH - AN APPROACH TO REDUCE GLYCEMIC INDEX FOR HEALTHY BREAD
}

\author{
Sunita Singh ${ }^{1}$ \\ sunitaiari@gmail.com \\ Shruti Sethi ${ }^{1}$ \\ docsethi@gmail.com \\ Sangeeta Gupta ${ }^{1}$ \\ sangeeta_21gupta@yahoo.in \\ Charanjit Kaur \\ charanjitkaur6@gmail.com \\ Ed Wood \\ Sourdoughs International \\ P.O. Box 670, Cascade, Idaho,USA, 83611 \\ edwood@sourdo.com \\ ${ }^{1}$ Division of Food Science and Post-harvest Technology \\ Indian Agricultural Research Institute \\ Pusa Campus, New Delhi, India, 110012
}

\begin{abstract}
The use of sourdough as the starter culture for bread making is one of the oldest processes in food fermentation and is very much prevalent in being used for the manufacture of various multigrain breads. The fermentation process of breads from mixed flours is one way, reported to reduce the glycemic index as compared to white bread. In this paper, we have discussed the use of (autochthonous) native culture vs pure culture use, in fermentation to prepare a starter culture sourdough by propagative fermentation. Since such a dough is incorporated in the sourdough bread making process (1:3), by the initial process of intermittent back-slopping (at intervals of 3.5 and 7 days) to propagate sourdough with a starter culture, as a part of the process, we observed the reduction in glycaemic index of the sourdough itself to as low as $G I=40$, at $3^{\text {rd }}$ day of fermentation when the pure consortium and at $5^{\text {th }}$ day of fermentation $G I=43$, when the native consortium was used. The sourdough process is thus an essential tool, aimed to make healthy breads, as it is incorporated as an ingredient in the process, to make sourdough bread.
\end{abstract}

Keywords: sourdough, multigrain bread, acidity, glycaemic index, hydrolysis index, starter culture.

DOI: $10.21303 / 2504-5695.2019 .00994$

\section{Introduction}

Healthy diets with preferences on multigrain breads, can contribute to low glycaemic indices. Since the glycaemic index (GI) is a measure, used to classify foods, according to their potential to raise blood glucose levels [1], low GI foods are preferred in diets. High glycaemic foods are responsible for weight gain and obesity [2-4] and high consumption of such products has often been associated to an increased risk of type 2 diabetes [5, 6] and cardiovascular diseases [4].

Preferences towards healthy diets can be possible, if they are culturally relevant, actionable and accessible to all that can help to promote robust health systems [7]. Thus, an important issue by adopting healthy food systems to halt the rise of obesity, prevalence of raised blood pressure and Type 2 diabetes, generated indirectly by consumption of high GI foods, can help to reduce such non-communicable diseases [8]. The nutritive, sensory, textural, and shelf life 
advantages of the sourdough technology in manufacture of baked products has been reported in baked goods as wheat and rye breads, crackers, pizza, multigrain and gluten-free products also [9-11].

Fermentation of dough, using an initially prepared sourdough (starter), allows biochemical changes that occur in the carbohydrate components of the flour with the use of starter, due to microbial fermentative metabolism of combined metabolic activity of lactic acid bacteria and yeasts [12], can decrease the glycaemic index in such baked goods. Under this context, the flat breads from dough fermented in age old ways, 'sourdough' are also available in India, known colloquially by traditional names such as pao, nan, tandoori roti, kulcha, khamiri roti, moghlai roti. They are also available in many other Asiatic parts of the world. In Germany sourdough bread as german vollkornbrot, is awholegrain seed bread $[13,14]$ from whole wheat flour, with characteristic taste and flavor. Even cinnamon rolls (sweet), and various other sourdough breads [15], and brown breads (Kurbiskernbrot), have a mention of the use of whole wheat flour and flours that are locally available. Sourdough breads involve a fermentation step with a starter culture. The starter can be available or propagated in the flour to as old as 100 years, as used fresh/dry as starters cultures or even propagated finally as dried starter cultures, for the purpose [15]. Such techniques can play a role in modification and improvement of bread quality so prepared [16] with sourdough. Also some brown breads that are available in the Indian market are sprinkled with grains on the bread, instead of using multigrain flour to bake the bread. There are known limitations to the use of whole wheat flour in breads (brown breads), which tend to make it crusty and hard [17]. Little is known about predicted glycaemic indices of the sourdoughs due to fermentation of the dough to be used for bread making. However the use of starter culture(s) to make the bread dough, improves its final texture [17], taste and flavor [18]. Most importantly these starters can provide resistance to contamination by other microorganisms and starters can also be maintained for decades [19]. The use of either a native flour microflora or known pure culture in flour, as starter culture, can allow differences in fermentation of dough, due to their capacity to utilize the available carbohydrates present in the flour. Since the process of leavening sourdough, consists of adding an active microbial consortium [yeast and lactic acid bacteria (LAB)] in the flour, it helps in its acidification [20]. The use of starter cultures in this study, provided information on the spontaneous vs known culture use, in sourdough bread making. The resulting sourdough was used to formulate multigrain sourdough bread. Thus the present work aimed to evaluate the use of two different starter cultures (native microflora or pure culture in fermented dough), that may change the glycaemic indices of sourdough at different stages of the fermentation. Also fermentation allowed biochemical changes that favorably worked towards low sugar release also. The results have generated interest to further this study in making it a profitable enterprise for sourdough bread, in a market demand that prefers healthy diet products. The work thus explored fermented dough (sourdough), as a possibility to formulate the multigrain bread, with an acceptable taste. Such components are also known to bring down gluten contents in bread. Bread fermenting with sourdough technology is not very new to Indian markets with available flatbreads. Hence, if this technology gets adopted it can very well be brought into healthy diet list in an Indian market.

\section{Materials and Methods}

\section{1. Raw material and dough preparation}

Flour used: The flour mix used was from different grains, namely, whole wheat, barley, ragi and sorghum were mixed in the proportion 80:10:5:5 (w/w) respectively. The proportions of these flours were decided, based on preliminary trials (not reported herein) suitable for bread making [21]. This multigrain mix flour was pasteurized for two consecutive days and used in two dough preparations. Two kinds of dough were prepared, based on two different starter culture doughs, used.

The starter culture preparation: Starter culture (used as fresh dough starter) was prepared by Sourdough Type II process [22], for propagating sourdough with starter culture from:

1) native microflora containing, sourdough starter, that was propagated in the flour naturally;

2) a sourdough starter, made and propagated with an added starter flour (dry, contained pure cultures for San Francisco bread), supplied by Sourdough International, Idaho, USA, as comple- 
mentary packets. The pure culture in flour, when supplied by Sourdough Int., was communicated as being the original culture [20,23], that was used for sanfrancisco bread, and used here in this study.

These two fermented doughs, were the starter culture doughs and also used for propagating mixed flour to higher volumes for bread. Thus, the starter dough and sourdough for bread were scaled up simultaneously. The mix that was propagated to higher volume starter culture dough contained starter dough and fresh dough in ratio 1:3 (starter culture dough: flour mix with water).

Process to propagate the sourdough by fermentation to a higher volume:

The propagation/up-scaling of the dough followed same ratio (1:3), each time the dough was needed in a higher volume, and left to ferment under room temperature $\left(21 \pm 3{ }^{\circ} \mathrm{C}\right)$, to a fermented dough mix, with back slopping technique after every $72 \mathrm{~h}$ intervals. Both the doughs were thus propagated by back-slopping technique on $0^{\text {th }}$ day, $3^{\text {rd }}$ day, $5^{\text {th }}$ day and $7^{\text {th }}$ day to reach a final volume of $1200 \mathrm{~g}$ dough (Fig. 1) in each case, for making sourdough bread [15] (Fig. 2). This was thus done to suit the requirement of making bread using the sourdough technology.

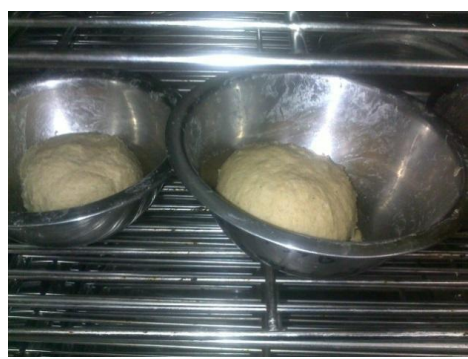

Fig. 1. Final sourdoughs for sourdough bread

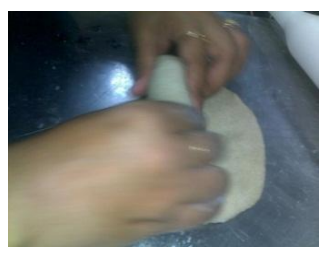

$a$

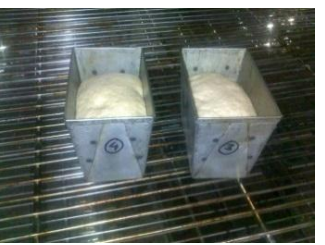

$b$

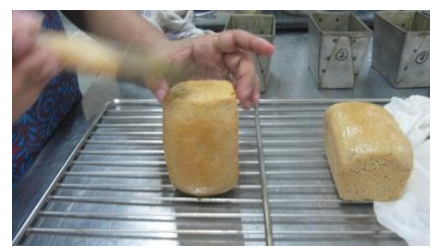

$c$

Fig. 2. Processing the sourdough to bread after kneading: $a$ - Dough shaping; $b$ - Loaf proofed; $c$ - Brushing oil on a baked bread loaf, to shine and prevent a loss of moisture (optional)

\section{2. Biochemical Analysis}

At each period of propagation, a sample $(20 \mathrm{~g})$ from the fermented dough was harvested and frozen at $0{ }^{\circ} \mathrm{C}$ for further analysis. The $\mathrm{pH}$, titratable acidity, total solid content of such fermented/ propagated dough (with native/pure starters) was determined by standard procedures. The $\mathrm{pH}$ was determined with a $\mathrm{pH}$ meter (Labman Scientific Instruments Model No LMPH 10) that was previously standardized. The total acidity was indicated from a titer value of a $10 \mathrm{~g}$ of sample that was diluted to $100 \mathrm{~mL}$ with distilled water and titrated with $0.1 \mathrm{~N} \mathrm{NaOH}$ with an indicator (phenolphthalein), to an endpoint of titration as a permanent light pink color that persisted for $30 \mathrm{sec}$, usually obtained at $\mathrm{pH}$ of 8.3 [24]. The acidity was expressed as a titer volume of alkali to neutralize the total acidity present.

The total solid contents were determined by heating a sample in an oven at $105{ }^{\circ} \mathrm{C}$ until a constant weight was obtained and calculated for percent solids, present in a sample, and calculated as below (1).

$$
\% \text { Solids }=\frac{\text { Wt of dry sample } \times 100}{\text { Wt of fresh sample }} .
$$

Since the propagated fermented dough was used to bake bread, we studied the glycaemic index (GI) of fermenting dough on $0,3,5$ and 7 days of fermentation. For this the reducing sugars 
[Total reducing sugars (TRS)] and total carbohydrates (TC) that were released in dough $(0,3,5$ and 7 days) were estimated $[25,26]$. An in-vitro dialysis procedure was followed [27], where a $5 \mathrm{~g}$ sample was homogenized in $20 \mathrm{~mL}$ of $0.1 \mathrm{M}$ potassium phosphate buffer solution ( $\mathrm{pH}$ 6.9) at $37.8^{\circ} \mathrm{C}$ in $100 \mathrm{~mL}$ Erlenmeyer flask. The system simulated the digestive tract environmental temperature. The sample $\mathrm{pH}$ was lowered to $\mathrm{pH} 1.5$ with $8 \mathrm{M} \mathrm{HCl}$, and digested with $1 \mathrm{~mL}$ pepsin enzyme (115 units) (Sigma-Aldrich). The sample was then placed in a water bath at $37.8^{\circ} \mathrm{C}$ for 30 min incubation with stirring with glass ball beads, to simulate peristalsis. Each sample was then buffered back to $\mathrm{pH} 6.9$ with $10 \% \mathrm{NaOH}$ solution and $1 \mathrm{~mL} \alpha$-amylase enzyme (16 units) (Sigma-Aldrich) added and left to digest for $10 \mathrm{~min}$ in the same water bath. All such digested sample contents in the flask were then transferred into a dialysis tubing (MWCO 14,000) $(250 \times 20 \mathrm{~mm}$ strips). The ends of the tubing bags were clipped and placed individually in flasks, containing $500 \mathrm{~mL}$ of phosphate buffer solution $\mathrm{pH}$ 6.9, under continuous and slow stirring (115 rpm), emulating intestinal movements. A dialysate volume of $30 \mathrm{~mL}$ was drawn out from the buffer solution, at every 30 minutes interval in the 4 hrs of dialysis and the volume was replaced with fresh buffer. Each of the so withdrawn dialyzed samples was placed in a boiling water bath for 5 minutes, quickly cooled and kept frozen until further analysis for total reducing sugars and total carbohydrates. Thus, these individually dialyzed samples of sourdough (that were sampled in different periods of being fermented), were analyzed for glucoses (as TRS) [25] and TC [26], using glucose as a standard and buffer (phosphate buffer solution $\mathrm{pH}$ 6.9), as a negative control of fermentation. The concentration of glucose (moles/L) vs time (0-180 min) was graphically analyzed for an area under curve (AUC) for each of the fermented samples. The rate of carbohydrate hydrolysis for hydrolysis index (HI) values was calculated as:

$$
\mathrm{HI}=\frac{\text { AUC (ferm sample) }}{\text { AUC (sample at } 0 \text { day })} \times 100 \text {. }
$$

The glycaemic index (GI), was then calculated from equation [28]:

$$
\mathrm{GI}=0 \cdot 862 \mathrm{HI}+8 \cdot 189 .
$$

The fermented samples were compared to the 0 day fermented starter dough for calculated GI.

\section{3. Enumeration of bacterial and yeast colonies}

The total counts of the pure dough starter cultures were enumerated by standard procedure [29] on Nutrient agar. The MRS agar and GPY medium (Hi-media) were used for enumeration by dilution plating of the sourdough samples for lactic bacteria and yeasts respectively. The native sourdough and pure culture samples were enumerated.

To isolate cultures from the pure culture, containing flour, used in the study, de- Mann Rogosa Sharpe agar (MRS agar) [30] and GPY media were used. The plates were incubated for 5 days and re-streaked if necessary to obtain pure culture isolates. The pure isolates were sub-cultured and stored for any further mass culture requirement.

\section{Results}

\section{1. Flours as an ingredient}

Blending wheat flour with other flours can bring additional advantages during SD preparations. As with sorghum as a flour in the blend for SD held significance during fermentation. This flour has a kafirin-rich protein matrix, usually not affected by proteolytic degradations. But during fermentations, soluble peptides are released from hydrolysed proteins that are taken up for the bacterial growth, from sorghum flour [31] which ultimately leads to the souring action in fermenting dough. On the other hand, on boiling/cooking of this flour (as in pasta), kafirins are converted into almost insoluble protein aggregates that are not accessible to disulphide-reducing agents. This reflects its importance in fermentation when present in sourdough versus unfermented flour (extruded) when used for pasta making. Nevertheless, each product has its own advantages, 
but still fermentation, plays a role in the dough, by releasing starch granules from such compact (kafirin-rich protein) matrices. On the other hand, the use of barley flour can help to give a rise in dough, by preventing a collapse of the loaf at proofing stage and also helps to give a good texture to the bread loaf [32].

\section{2. Sourdough starter inoculums/ cultures}

In our study, the doughs from both the fermented samples showed countable colonies after $10^{6}$ times dilution of sample. The native culture fermented dough showed $5 \times 10^{8} \mathrm{cfu} / \mathrm{g}$ with 5-6 different morphological colony types. The pure dough however showed a gummy yeast culture on the glucose peptone yeast extract agar (GPY) medium that lost the gumminess after repeated streaking. Lactic acid bacteria, isolated on MRS agar medium [30], and yeast culture, isolated on GPY medium (by incubation at $30{ }^{\circ} \mathrm{C}$ ), from the dough. They have not been taken up for detailed studies. The pure cultures are reported to be as used in bakery previously [20;23]. Previously it was reported [33] that total bacterial counts of fourteen sourdough samples to be in the range of 5.97 to $9.57 \log \mathrm{cfu} \mathrm{g}^{-1}$.

Since sourdough that has constant properties (e. g., acidification, leavening capacity) is mature, we considered the propagated sourdough (Fig. 3, a) as mature. This dough had a pleasing flavor and odor and was thus used as a starter for making multigrain bread (Fig. 3, b).

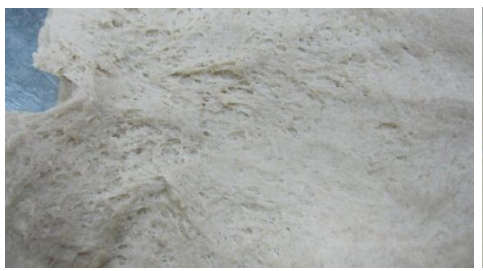

$a$

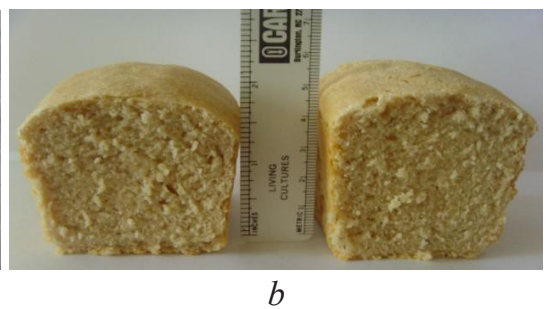

Fig. 3. Dough proof and loaf proof: $a$-Flattening dough in shaping step, observing mature fermented sourdough; $b$ - Multigrain bread made from the sourdough showing evenly distributed holes and a soft texture

The total counts of this pure sourdough starter were $7 \times 10^{7} \mathrm{cfu} . \mathrm{g}^{-1}$ on the nutrient agar medium. At this stage, lactic acid bacteria (on MRS agar) reached values as high as $53 \times 10^{8} \mathrm{cfu}^{-1}$. Similar values have also reported earlier for mature dough $9.0 \log \mathrm{cfu} . \mathrm{g}^{-1}$ [6].

\section{3. Changes in the $\mathrm{pH}$, titratable acidity and total solid content of the fermented dough}

Changes in $\mathrm{pH}$, titratable acidity (\%) and total solid (\%) content with respect to fermentation time of the leavened doughs using two different starters are shown (Table 1). As is evident, there was a continuing decline in the $\mathrm{pH}$ of the dough, fermented with the native microflora, till $7^{\text {th }}$ day of propagation, showing the lowest $\mathrm{pH}$ value of 5.04 .

In contrast, fermentation of dough with the pure starter culture led to maintenance of $\mathrm{pH}$ throughout the fermentation period and a gradual increase in titratable acidity. There seemed to be a $\mathrm{pH}$ buffering stability that persisted at $\mathrm{pH} \mathrm{6.2,} \mathrm{along} \mathrm{with} \mathrm{the} \mathrm{increasing} \mathrm{titratable} \mathrm{acidity} \mathrm{level} \mathrm{in}$ the dough, at each propagation stage (Table 1).

A microbiota development for sourdough is known to be a deciding factor in fermentation, especially when using pure culture dough starter [34]. The starter dough favours the growth of lactobacilli over the yeast growth, a characteristic feature, when $\mathrm{pH}$ values are observed to be $>4.5$ in the final sourdough, used for bread, and L. sanfranciscensis, in particular, does not grow below $\mathrm{pH} 3.8$ [22]. Such a $\mathrm{pH}$ environment of sourdoughs, formed after $7^{\text {th }}$ day, indicated predominance of lactic bacterial activity. The dough, prepared with the native starter culture, also showed comparable $\mathrm{pH}$ values. Thus, a dough environment can be a decisive growth- limiting factor, for the organisms in sourdough.

A similar reduction in $\mathrm{pH}$ of sourdoughs has been reported by others (35), in wheat-legume sourdough, chickpea sourdough and lentil sourdough during the 10 days back-slopping technique, 
as also with a corresponding increase in titratable acidity. Microbiota that develops in the changing $\mathrm{pH}$ of sourdough may be a reason to influence the final dough of a different starter culture. The mature Italian sourdoughs have $\mathrm{pH}$ from 3.70 to 4.28 in bread [6]. This technology still requires better insights into the genetic and phenotypic diversity of strains, to exploit them further. In previous studies, it has been shown that sourdough fermentations, carried out in the laboratory with flour as the sole non-sterile ingredient, harbor more different species diversity than artisan sourdoughs, prepared in a bakery, with respect to both LAB and yeast species [36-38]. Thus, preparing sourdough with the pure culture consortium with favorable metabolism can help to reduce the sugar release as required for a low GI, perhaps when the sourdough as a constituent, is used for baking it into bread.

\section{Table 1}

Changes in $\mathrm{pH}$, Titer for neutralizing acid and total solids (\%) of the sourdoughs after three, five and seven days of propagation

\begin{tabular}{|c|c|c|c|}
\hline Sourdough sample (days after propagation) & pH & Vol of $\mathrm{NaOH}(0.1 \mathrm{~N})(\mathrm{mL} / 10 \mathrm{~g}$ dough) & Total solids (\%) \\
\hline \multicolumn{4}{|c|}{ Native } \\
\hline 0 day & 6.61 & 14.48 & 43.360 \\
\hline $3^{\text {rd }}$ day & 5.71 & 15.12 & 39.264 \\
\hline $5^{\text {th }}$ day & 5.70 & 14.42 & 42.504 \\
\hline $7^{\text {th }}$ day & 5.04 & 14.75 & 41.032 \\
\hline \multicolumn{4}{|c|}{ Pure } \\
\hline 0 day & 6.90 & 2.97 & 49.522 \\
\hline $3^{\text {rd }}$ day & 5.98 & 8.71 & 54.795 \\
\hline $5^{\text {th }}$ day & 6.21 & 9.87 & 42.280 \\
\hline $7^{\text {th }}$ day & 6.20 & 9.82 & 45.286 \\
\hline
\end{tabular}

Now observing the acidity in fermentation of dough with the pure starter culture, an increase at $3^{\text {rd }}$ day, intermediate phase, is an evidence of the presence of yeasts and lactobacilli that increased in propagation of the dough. They are metabolically active [39] during propagation and can hydrolyze carbohydrates (HI, Table 2) in the fermenting dough. Such changes in the sourdoughs were similar to those, reported in earlier studies [12, 28].

\section{Table 2}

Release of reducing sugars and carbohydrates from sourdough samples after in vitro digestion with pepsin and amylase

\begin{tabular}{|c|c|c|c|}
\hline $\begin{array}{l}\text { S. No. (Days of fermentation } \\
\text { after inoculum added") }\end{array}$ & $\begin{array}{l}\text { Maximal release of TRS } \\
\text { (mg \%) (in digestion time, } h \text { ) }\end{array}$ & Hydro lysis index & Glyce mic index \\
\hline \multicolumn{4}{|c|}{ Native SD as Inoculum propagated in flour mix } \\
\hline $1(0)$ & $53.28(3.5)$ & 100.00 & 94.4 \\
\hline $2(3)$ & $46.64(3)$ & 90.33 & 86.1 \\
\hline $3(5)$ & $26.68(3)$ & 40.84 & 43.4 \\
\hline $4(7)$ & $18.22(3)$ & 31.12 & 35.0 \\
\hline \multicolumn{4}{|c|}{ Pure SD as Inoculum propagated in flour mix } \\
\hline $5(0)$ & $32.55(4)$ & 100.00 & 94.4 \\
\hline $6(3)$ & $14.48(3.5)$ & 36.74 & 39.9 \\
\hline $7(5)$ & $11.66(4)$ & 35.16 & 38.5 \\
\hline $8(7)$ & $11.66(4)$ & 27.86 & 32.2 \\
\hline
\end{tabular}

Note: * - Inoculum was also propagated for a period of 3 days initially before adding in to flour mixes for further propagation;

s - Total reducing sugars are expressed as glucose 
Comparing the pure culture to native culture dough fermentations, all the sourdoughs, developed by pure culture fermentations, showed much lower titratable acidity, as compared to the native culture fermented counterparts till the last period, under propagation. To note, the sample propagated by pure cultures, had lower final acidity $(9.82 \%)$ than that, propagated by the native culture dough $(14.75 \%)$, on the $7^{\text {th }}$ day.

The solid content in the samples, fermented with the native culture dough and pure culture dough, was $41 \%$ and $48 \%$, respectively. Thus, the native micro-flora and pure culture (of lactic acid bacteria and yeast), propagated in the dough, were the reason for such varying levels of $\mathrm{pH}$ and acidity in the fermentations. Hence, pure culture fermentations are always a preference to spontaneous fermentations, wherein we can obtain fermented conditions more suitable to a taste (less sour), more liked in a product like bread. Since this was sourdough fermentation, the acidity and $\mathrm{pH}$, observed in dough, fermented with the native culture, showed $\mathrm{pH}$ and TA changes towards making the dough more acidic and perhaps sour, fermentation with the pure culture would be far much a preferred starter for such products (bread).

The other advantages of sourdough fermentations are for the lactic and acetic acids, produced in the medium to resist contamination by other microorganisms [19] and also for improved flavors, as in wheat breads [38]. The use of the pure culture in the multigrain sourdough yielded characteristically pleasant and fruity aromas in the dough as compared to native culture sourdough. According to previous report [40], whole wheat flour sourdough bread, enriched with oat fibre, showed more pronounced acidulous smell and taste, and an intense and more appreciated aroma with respect to normal breads, owing to the lactic acid bacteria, growing in the sourdough.

The analysis showed an overall status of fermentable ingredients on GI of dough which may have an influence on the ultimate low sugar diet requirement from consuming bread. The sourdough mix when propagated and fermented till a 7 day period showed maximum total reducing sugars (TRS) at 0 day in sourdough (53 mg \%) that reduced with fermentation to $47 \mathrm{mg} \%$, $27 \mathrm{mg} \%$ \& $18.22 \mathrm{mg} \%$ at $3^{\text {rd }}$ and $5^{\text {th }} \& 7^{\text {th }}$ day after propagated fermentation with the native flora respectively (Table 2). All these fermented samples showed the maximal release of these sugars after $3 \mathrm{~h}$ of enzymatic digestion (in vitro). Interestingly, in contrast to the higher release of reducing sugars in case of the samples, fermented with the natively present micro-flora of flour, there was a steady release of reducing sugars in the samples, fermented with pure cultures and known sourdough inoculums, that released $14 \mathrm{mg} \%$ (3 day) to $12 \mathrm{mg} \%$ ( $^{\text {th }}$ and $7^{\text {th }}$ day) from 33 $\mathrm{mg} \%$ of TRS, present initially in the inoculated sample at 0 day. Again, digestion of carbohydrates showed less release into the solution when the flour was fermented with sourdough of known pure cultures as compared to the samples, fermented with the native microflora (Table 4). The reduction in hydrolysis indices of the samples was also directly related to incremental days of fermentation. This pointed to a potential lowering of glycaemic index due to fermentation. The sourdough from a pure culture as inoculum was a better potential starter in reducing glycaemic index of sourdough in 3 days only as compared to 5 days of fermentation with the native microflora.

\section{In vitro digestibility of carbohydrates in fermented dough}

Sourdough, propagated with the native culture, showed randomness in breakdown of the complex polysaccharides during the 3 h digestion period (Fig. 4) in the propagated samples at different time intervals. The release of reducing sugars was not uniform as monitored over 30 minutes regular intervals of invitro digestion.

On the other hand, the dough propagated using the pure culture, sampled at the different propagation periods $(0,3,5$ and 7 days), showed a gradual and progressive increase in the reducing sugars with the increase in digestion period (Fig. 5). The maximal sugars released were $46 \mathrm{mg} \%$ in the native sourdough sample ( $3 \mathrm{rd}$ day, $3 \mathrm{~h}$ digestion period) as compared to $14.48 \mathrm{mg} \%$ in the pure culture sourdough sample (3rd day, $3.5 \mathrm{~h}$ digestion period) (Table 2).

In the sourdough, fermented by native cultures, the reducing sugars ( 3 days after fermentation), released after $3 \mathrm{~h}$ digestion, were about $80 \%$ higher in dough as compared to sourdough fermented with pure cultures. The pure culture propagated sourdough showed a sharp $36 \%$ drop in the total reducing sugars, released post 3 days fermentation (Fig. 3, 4). At the $7^{\text {th }}$ day of fermen- 
tation the sourdough with the native microflora released about $58 \%$ higher total reducing sugar content as compared to the pure culture propagated dough. The higher titratable acidity and also the presence of enzymes maybe attributed to such an observation [41]. A low release of sugars is the preference for dietary GI. Thus, the pure culture propagated sourdough that showed the slow release of sugars (TRS) is the dough, preferred for use in making multigrain bread (Fig. 2, b), indicating the low GI that would also be present in bread.

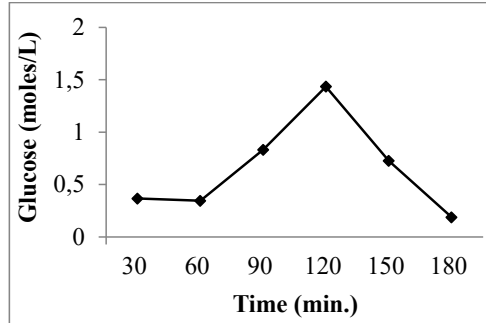

$a$

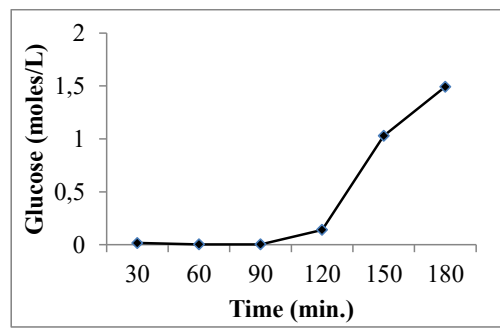

$c$

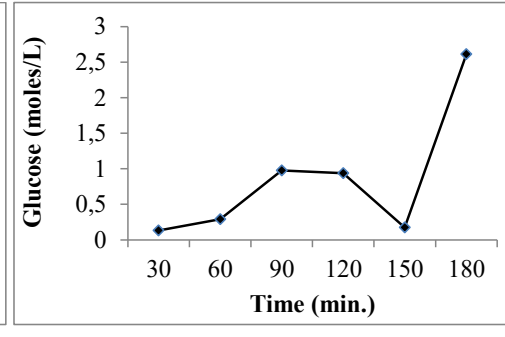

$b$

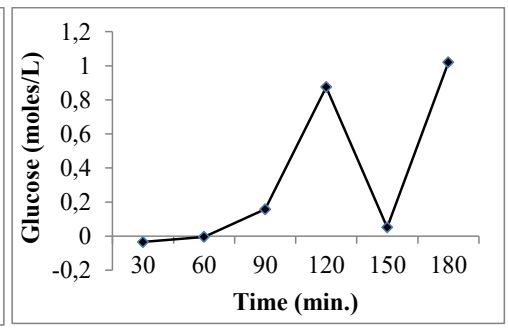

$d$

Fig. 4. Total reducing sugars (moles/L) released in sour dough propagated by native cultures for different intervals: $a-0$ day; $b-3$ days; $c-5$ days; $d-7$ days

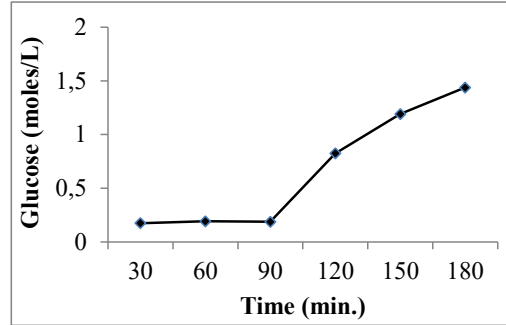

$a$

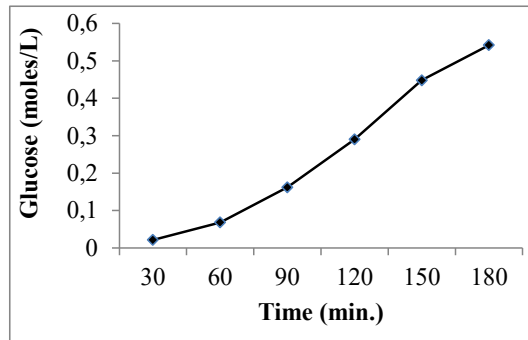

$c$

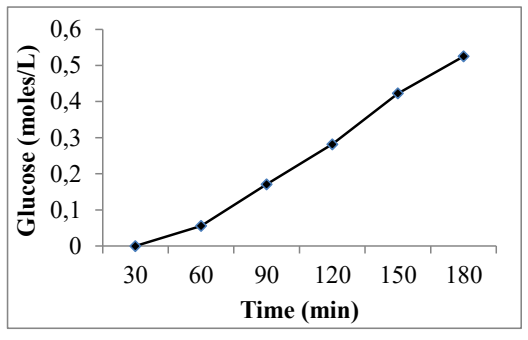

$b$

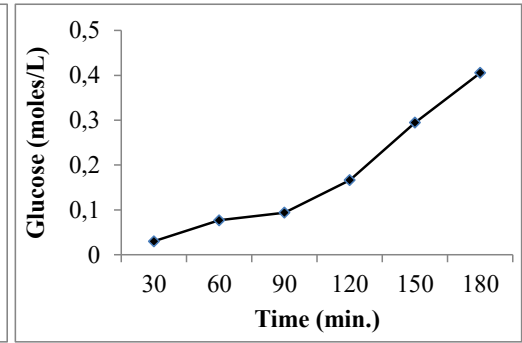

$d$

Fig. 5. Total reducing released in sour dough propagated by pure cultures for different intervals: $a-0$ day; $b-3$ days; $c-5$ days; $d-7$ days

The hydrolysis indices (of carbohydrates) and predicted GI of sourdough at different intervals of propagation, using the native and pure microflora, are shown in Table 2 . The rate of in vitro carbohydrate hydrolysis was used for GI as it is considered to be a presumptive measure of the GI 
in healthy subjects [42]. The hydrolysis index (HI) is dependent on the total release of sugars (TRS) in the full period of digestion. A higher HI indicates higher AUC for sugars in dialysates. An overall low rate of hydrolysis up to 3 days could be a reason, explained by the presence of the native microflora (spontaneous fermentation), whereas pure culture fermentations helped in higher rate of hydrolysis at 3 days. The corresponding, GI values were much lowered in the dough, developed by pure culture propagation, after 3 days of fermentation. In case of native culture propagation, the low GI values were obtained only after 5 days of fermentation in the dough (Table 2).

\section{Conclusion}

Dough fermentation creates a matrix, formed by the entrapment of gas bubbles, due to yeasts in fermentative stage along with dough expansion (leavening) and acidity development due to added microbial inocula. The leavening step enhances the surface, exposed to enzymatic activity. L. sanfranciensis, the lactic culture with yeast strain, in the pure starter culture consortium, as supplied, was used in this study. L. sanfranciscensis is also known for its ability to produce an enzyme, glutathione reductase (GshR). This enzyme can reduce the sulfhydryl compounds, present in wheat flour. This culture then helps to maintain a required redox homeostasis in fermentation due to its GshR activity and serves in disulphide exchange reactions in wheat doughs. The sulfhydryl compounds, which undergo a disulfide-sulfhydryl interchange with other low-molecular-weight thiol compounds, can cause cleavage or reformations of disulfide bonds in wheat dough. Such interactions lead to formation of glutenin macropolymers in wheat doughs that then determine a dough rheology, and its gas retention. All such changes influence the resulting consistency of dough [43]. The presence of such lactic starters, thus contribute to beneficial effects in sourdough fermentation and in bread texture. Thus, bread volume and texture is hence determined both by the flour and starter culture used.

Now considering the aspect of healthy diet, the sourdough was a source of reduced sugars in fermented flour (sourdough) itself. Also that the fermentable carbohydrates that remain in the bread even after baking also can affect the food's GI and can also have a potential to regulate postprandial responses to a second meal effect, by reducing non-esterified fatty acids (NEFA) competition for glucose disposal and, to a minor extent, they also affect the intestinal motility [44]. Low postprandial blood glucose is associated with a low risk of metabolic diseases (diabetes).

Thus, the sourdough technology has beneficial effects and can provide healthy diet food in the form of sourdough multigrain bread.

To the best of our knowledge, the application of sourdough to the fermentation of wheat-sorghum-barley mix sourdough in bread making has never been investigated. This study gives a comprehensive and comparative view of the effect of fermentation on the development of sourdough, by a spontaneous fermentation (native flora in dough) as compared to fermentation with known starter (pure microbiota) culture dough. Sourdough, prepared in making bread, can also be expected to have reduced GI values and are yet to be confirmed though. However, such GI values are an interesting part of this investigation that has never been explored earlier. An acceptable taste and softer texture (details not reported here) (Fig. 6, $\boldsymbol{a}$ ) as compared to similar brown bread, available in the market of Austria, prepared by sourdough fermentation (Fig. 6, b, $c$ ) had a very hard inner texture.

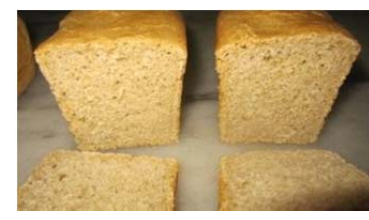

$a$

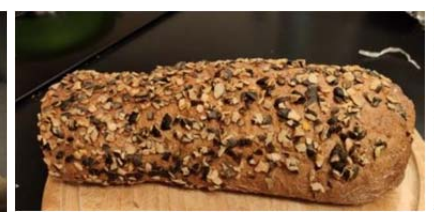

$b$

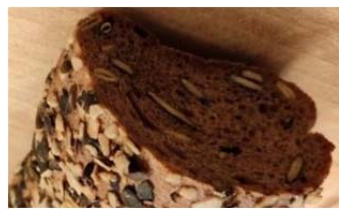

$c$

Fig. 6. Sourdough breads showing color and inner texture of bread: $a$-Sourdough multigrain bread (this study); $b$ - Brown bread (available in Vienna, Austria), see the hard outer crust; $c$-harder inner texture

Reports are available on the different kinds of flours that can reduce GI values of gluten free breads [27]. The GIs so reported were in the range of 69 to 95 which are high, but lower than white 
breads. Low GI bread from sourdough, made from such low GI sourdough starters, is the next important component to be accomplished for further inclusions in healthy diets. The use of the pure starter culture in propagation of sourdough is thus emphasized by this study. This technique can also be used to enrich the functional quality with bioactive compounds, for health (anti- inflammatory activity, from black carrot color extracts substitution in wheat flour as 7-8 \%, for bread [45-47]. By adding such ingredients at sourdough stage, we can also increase digestibility of bioactive anthocyanin compounds in black carrots [48]. The results of this study will be helpful to choose specific starter cultures and specific fermentation time of sourdough starter, to develop low glycaemic multigrain breads.

Multigrain breads, prepared by the use of the sourdough process, can help to improve the bread volume, crumb structure [49-52], flavour [18], nutritional values [49, 53, 54] and shelf-life [49, 55-58].

Henceforth this technology can have multifaceted advantages towards bread, with better textural properties along with health benefits towards lifestyles disorder diseases, as type 2 diabetes, that is widespread in the present world. To have a sourdough bread-making technology in place with its benefits as above, will be a direction towards listing healthy diet foods.

One of the limitations in this technology is the mass multiplication of the starter and availability of a pure starter culture, especially in India. The feasibility of using a starter culture from an already available manufacturing/production unit, as supplied by Sourdough Int. (in this study), shows that this technology is an adoptable technology. The technology must have hygiene certifications (HACCP), necessary both for a starter culture production unit as well as for the bread-making production unit. Attention also needs to be drawn on the use of appropriate starters. Some of the lactic bacteria are known to produce biogenic amines, that can result into toxicological problems, if such foods contain relatively high levels of these compounds (especially tyramine and histamine), and consumed [59]. However, the starters, used in this study, produced none or negligible levels of biogenic amines, as checked, in vitro for histamine and putrescine [60].

\section{Acknowledgement}

The authors are thankful to the Ministry of Food Processing Industries (MOFPI, Government of India), for funding this work. The authors are also thankful to the and Director, IARI, New Delhi and Head, Division of Food Science and Technology for providing the necessary facilities. We are much thankful to Dr Anil Dahuja, Division of Biochemistry in providing certain lab facilities without any hesitation.

\section{References}

[1] Whitney, E. N., Rolfes, S. R. (2002). Understanding Nutrition. Wadsworth, 920.

[2] Ludwig, D. S. (2000). Dietary Glycemic Index and Obesity. The Journal of Nutrition, 130 (2), 280S-283S. doi: https://doi.org/ $10.1093 / \mathrm{jn} / 130.2 .280 \mathrm{~s}$

[3] Liu, S., Willett, W. C., Stampfer, M. J., Hu, F. B., Franz, M., Sampson, L. et. al. (2000). A prospective study of dietary glycemic load, carbohydrate intake, and risk of coronary heart disease in US women. The American Journal of Clinical Nutrition, 71 (6), 1455-1461. doi: https://doi.org/10.1093/ajcn/71.6.1455

[4] Foster-Powell, K., Holt, S. H., Brand-Miller, J. C. (2002). International table of glycemic index and glycemic load values: 2002. The American Journal of Clinical Nutrition, 76 (1), 5-56. doi: https://doi.org/10.1093/ajen/76.1.5

[5] Rondini, E. A., Bernink, M. R. (2007). Ontario White Bean Producers. Nutrition and health. Available at: http://ontariobeans.on.ca/

[6] Minervini, F., Di Cagno, R., Lattanzi, A., De Angelis, M., Antonielli, L., Cardinali, G. et. al. (2011). Lactic Acid Bacterium and Yeast Microbiotas of 19 Sourdoughs Used for Traditional/Typical Italian Breads: Interactions between Ingredients and Microbial Species Diversity. Applied and Environmental Microbiology, 78 (4), 1251-1264. doi: https://doi.org/10.1128/aem.07721-11

[7] Khetrapal, S. P. (2018). How healthy food systems can reduce noncommunicable diseases: India and neighbours are acting, but more must be done. Available at: https://timesofindia.indiatimes.com/blogs/toi-edit-page/how-healthy-food-systems-canreduce-noncommunicable-diseases-india-and-neighbours-are-acting-but-more-must-be-done/

[8] Director-General's Office. WHO. Available at: https://www.who.int/dg/regional_directors/poonam-khetrapal-singh/en/

[9] Arendt, E. K., Moroni, A., Zannini, E. (2011). Medical nutrition therapy: use of sourdough lactic acid bacteria as a cell factory for delivering functional biomolecules and food ingredients in gluten free bread. Microbial Cell Factories, 10 , S15. doi: https://doi.org/10.1186/1475-2859-10-s1-s15 
[10] De Vuyst, L., Vrancken, G., Ravyts, F., Rimaux, T., Weckx, S. (2009). Biodiversity, ecological determinants, and metabolic exploitation of sourdough microbiota. Food Microbiology, 26 (7), 666-675. doi: https://doi.org/10.1016/j.fm.2009.07.012

[11] Gobbetti, M. (1998). Interactions between lactic acid bacteria and yeasts in sourdoughs. Trends in Food Science and Technology, 9, 267-274.

[12] Gänzle, G. G., Ehmann, M., Hammes, W. P. (1998). Modeling of Growth of Lactobacillus sanfranciscensis and Candida milleri in Response to Process Parameters of Sourdough Fermentation. Applied and Environmental Microbiology, 64 (7), $2616-2623$.

[13] The home of great sourdough-everything you need to bake your own amazing sourdough. Available at: https://foodbodsourdough.com/

[14] Sprouted Vollkornbrot With Seeds. Available at: https://www.abreaducation.com/blog/2012/08/sprouted-vollkornbrot

[15] Wood, E., Wood, J. (2011). Classic Sourdoughs. Ten Speed Press, 38-39.

[16] Pyler, E. J., Gorton, L. (2016). Pyler says: The story of San Francisco sourdough. Available at: https://www.bakingbusiness. com/articles/44795-pyler-says-the-story-of-san-francisco-sourdough

[17] Development of Multigrain Bread Having Low GI Using Sourdough Technology. In IARI Annual Report (2014-2015). Available at: https://www.iari.res.in/files/Publication/annual_report/Annual-Report_2014-15.pdf

[18] Thiele, C., Gänzle, M. G., Vogel, R. F. (2002). Contribution of Sourdough Lactobacilli, Yeast, and Cereal Enzymes to the Generation of Amino Acids in Dough Relevant for Bread Flavor. Cereal Chemistry Journal, 79 (1), 45-51. doi: https://doi.org/ 10.1094/cchem.2002.79.1.45

[19] Kline, L., Sugihara, T. F. (1971). Microorganisms of the San Francisco Sour Dough Bread Process. II. Isolation and Characterization of undescribed bacterial species responsible for the souring activity. Applied Microbiology, 21 (3), 459-465.

[20] Kline, S., Sugihara, T. F., McCready, L. B. (1970). Nature of the San Francisco sour dough French bread process. I. Mechanics of the process. Bakers Digest, 44 (2), 48.

[21] Kaur, C. (2014). Project Annual Report 2014. Development of phenolic enriched multigrain bread with improved functional quality. A Ministry of Food Processing Industries funded MOFPI Project No SERB/MOFPI/006/2013.

[22] Siepmann, F. B., Ripari, V., Waszczynskyj, N., Spier, M. R. (2017). Overview of Sourdough Technology: from Production to Marketing. Food and Bioprocess Technology, 11 (2), 242-270. doi: https://doi.org/10.1007/s11947-017-1968-2

[23] Ottogalli, G., Galli, A., Foschino, R. (1996). Italian bakery products obtained with sourdough: Characterization of the typical microflora. Advances in Food Sciences, 18 (5/6), 131-144.

[24] Lland Patrick, O. A. M., Bruer, N., Edwards, G., Caloghiris, S., Eric, W. (2013). Chemical Analysis of Grapes and Wine: Techniques and Concepts 2nd Edition. Patrick Iland Wine Promotions Pty Ltd., 114.

[25] Miller, G. L. (1959). Use of Dinitrosalicylic Acid Reagent for Determination of Reducing Sugar. Analytical Chemistry, 31 (3), 426-428. doi: https://doi.org/10.1021/ac60147a030

[26] Turula, V. E., Gore, T., Singh, S., Arumugham, R. G. (2010). Automation of the Anthrone Assay for Carbohydrate Concentration Determinations. Analytical Chemistry, 82 (5), 1786-1792. doi: https://doi.org/10.1021/ac902664x

[27] Wolter, A., Hager, A.-S., Zannini, E., Arendt, E. K. (2013). In vitro starch digestibility and predicted glycaemic indexes of buckwheat, oat, quinoa, sorghum, teff and commercial gluten-free bread. Journal of Cereal Science, 58 (3), $431-436$. doi: https://doi.org/10.1016/j.jcs.2013.09.003

[28] Brennan, C. S., Tudorica, C. M. (2008). Evaluation of potential mechanisms by which dietary fibre additions reduce the predicted glycaemic index of fresh pastas. International Journal of Food Science \& Technology, 43 (12), 2151-2162. doi: https:// doi.org/10.1111/j.1365-2621.2008.01831.x

[29] Collins, C. H., Lyne, P. M. (1964). Collins and Lyne's Microbiological Methods. Butterworth-Heinemann Pub., 465.

[30] De Man, J. C., Rogosa, M., Sharpe, M. E. (1960). A medium for the cultivation of lactobacilli. Journal of Applied Bacteriology, 23 (1), 130-135. doi: https://doi.org/10.1111/j.1365-2672.1960.tb00188.x

[31] Elkhalifa, A. E. O., Bernhardt, R., Bonomi, F., Iametti, S., Pagani, M. A., Zardi, M. (2005). Fermentation modifies protein/ protein and protein/starch interactions in sorghum dough. European Food Research and Technology, 222 (5-6), 559-564. doi: https://doi.org/10.1007/s00217-005-0124-9

[32] Hart, M. R., Graham, R. P., Gee, M., Morgan, A. I. (1970). Bread from sorghum and barley flours. Journal of Food Science, 35 (5), 661-665. doi: https://doi.org/10.1111/j.1365-2621.1970.tb04837.x

[33] Gül, H., Özçelik, S., Sağdıç, O., Certel, M. (2005). Sourdough bread production with lactobacilli and S. cerevisiae isolated from sourdoughs. Process Biochemistry, 40 (2), 691-697. doi: https://doi.org/10.1016/j.procbio.2004.01.044

[34] Sugihara, T. F., Leo, K., Miller, M. W. (1971). Microorganisms of the San Francisco Sour Dough Bread Process I. Yeasts Responsible for the Leavening Action. Applied Microbiology, 21 (3), 456-458.

[35] Rizzello, C. G., Calasso, M., Campanella, D., De Angelis, M., Gobbetti, M. (2014). Use of sourdough fermentation and mixture of wheat, chickpea, lentil and bean flours for enhancing the nutritional, texture and sensory characteris- 
tics of white bread. International Journal of Food Microbiology, 180, 78-87. doi: https://doi.org/10.1016/j.ijfoodmicro. 2014.04.005

[36] Vrancken, G., De Vuyst, L., Van der Meulen, R., Huys, G., Vandamme, P., Daniel, H.-M. (2010). Yeast species composition differs between artisan bakery and spontaneous laboratory sourdoughs. FEMS Yeast Research, 10 (4), 471-481. doi: https:// doi.org/10.1111/j.1567-1364.2010.00621.x

[37] Weckx, S., Van der Meulen, R., Maes, D., Scheirlinck, I., Huys, G., Vandamme, P., De Vuyst, L. (2010). Lactic acid bacteria community dynamics and metabolite production of rye sourdough fermentations share characteristics of wheat and spelt sourdough fermentations. Food Microbiology, 27 (8), 1000-1008. doi: https://doi.org/10.1016/j.fm.2010.06.005

[38] Hansen, A., Hansen, B. (1996). Flavour of sourdough wheat bread crumb. Zeitschrift Fur Lebensmittel-Untersuchung Und Forschung, 202 (3), 244-249. doi: https://doi.org/10.1007/bf01263548

[39] Schulz, A. (1972). Der EinflussorganiscerSauren auf die Vergarungverschiedener Z. Getr Mehl Brot, 26, 129-33.

[40] De Angelis, M. (2003). Phytase activity in sourdough lactic acid bacteria: purification and characterization of a phytase from Lactobacillus sanfranciscensis CB1. International Journal of Food Microbiology, 87 (3), 259-270. doi: https://doi.org/10.1016/ s0168-1605(03)00072-2

[41] John, R. P., G.S., A., Nampoothiri, K. M., Pandey, A. (2009). Direct lactic acid fermentation: Focus on simultaneous saccharification and lactic acid production. Biotechnology Advances, 27 (2), 145-152. doi: https://doi.org/10.1016/j.biotechadv.2008.10.004

[42] Åkerberg, A., Liljeberg, H., Björck, I. (1998). Effects of Amylose/Amylopectin Ratio and Baking Conditions on Resistant Starch Formation and Glycaemic Indices. Journal of Cereal Science, 28 (1), 71-80. doi: https://doi.org/10.1006/jcrs.1997.0173

[43] Jansch, A., Korakli, M., Vogel, R. F., Ganzle, M. G. (2007). Glutathione Reductase from Lactobacillus sanfranciscensis DSM20451T: Contribution to Oxygen Tolerance and Thiol Exchange Reactions in Wheat Sourdoughs. Applied and Environmental Microbiology, 73 (14), 4469-4476. doi: https://doi.org/10.1128/aem.02322-06

[44] Brighenti, F., Benini, L., Del Rio, D., Casiraghi, C., Pellegrini, N., Scazzina, F. et. al. (2006). Colonic fermentation of indigestible carbohydrates contributes to the second-meal effect. The American Journal of Clinical Nutrition, 83 (4), $817-822$. doi: https://doi.org/10.1093/ajen/83.4.817

[45] Charanjit, K., Singh, Sunita, Rudra Shalini, G., Shruti, S. (2015). Final Report of Project. Development of phenolic enriched multigrain bread with improved functional quality. A Ministry of Food Processing Industries funded Project No SERB/ MOFPI/006/2013.

[46] Gobbetti, M., Rizzello, C. G., Di Cagno, R., De Angelis, M. (2014). How the sourdough may affect the functional features of leavened baked goods. Food Microbiology, 37, 30-40. doi: https://doi.org/10.1016/j.fm.2013.04.012

[47] Lopez, H. W., Duclos, V., Coudray, C., Krespine, V., Feillet-Coudray, C., Messager, A. et. al. (2003). Making bread with sourdough improves mineral bioavailability from reconstituted whole wheat flour in rats. Nutrition, 19 (6), 524-530. doi: https:// doi.org/10.1016/s0899-9007(02)01079-1

[48] Park, S., Kang, S., Jeong, D.-Y., Jeong, S.-Y., Park, J. J., Yun, H. S. (2015). Cyanidin and malvidin in aqueous extracts of black carrots fermented with Aspergillus oryzae prevent the impairment of energy, lipid and glucose metabolism in estrogendeficient rats by AMPK activation. Genes \& Nutrition, 10 (2). doi: https://doi.org/10.1007/s12263-015-0455-5

[49] Arendt, E. K., Ryan, L. A. M., Dal Bello, F. (2007). Impact of sourdough on the texture of bread. Food Microbiology, 24 (2), 165-174. doi: https://doi.org/10.1016/j.fm.2006.07.011

[50] Clarke, C. I., Schober, T. J., Arendt, E. K. (2002). Effect of Single Strain and Traditional Mixed Strain Starter Cultures on Rheological Properties of Wheat Dough and on Bread Quality. Cereal Chemistry Journal, 79 (5), 640-647. doi: https://doi.org/ 10.1094/cchem.2002.79.5.640

[51] Corsetti, A., Gobbetti, M., De Marco, B., Balestrieri, F., Paoletti, F., Russi, L., Rossi, J. (2000). Combined Effect of Sourdough Lactic Acid Bacteria and Additives on Bread Firmness and Staling. Journal of Agricultural and Food Chemistry, 48 (7), 3044-3051. doi: https://doi.org/10.1021/jf990853e

[52] Crowley, P., Schober, T., Clarke, C., Arendt, E. (2002). The effect of storage time on textural and crumb grain characteristics of sourdough wheat bread. European Food Research and Technology, 214 (6), 489-496. doi: https://doi.org/10.1007/s00217002-0500-7

[53] Liljeberg, H., Bjorck, I. (1994). Bioavailability of starch in bread products. Postprandial glucose and insulin responses in healthy subjects and in vitro resistant starch conten. European Journal of Clinical Nutrition, 48 (3), 151-163.

[54] Liljeberg, H. G. M., Lonner, C. H., Bjorck, I. M. E. (1995). Sourdough fermentation or addition of organic acids or corresponding salts to bread improves nutritional properties of starch in healthy humans. Journal of Nutrition, 125 (6), 1503-1511.

[55] Corsetti, A., Gobbetti, M., Rossi, J., Damiani, P. (1998). Antimould activity of sourdough lactic acid bacteria: identification of a mixture of organic acids produced by Lactobacillus sanfrancisco CB1. Applied Microbiology and Biotechnology, 50 (2), 253-256. doi: https://doi.org/10.1007/s002530051285 
[56] Lavermicocca, P., Valerio, F., Evidente, A., Lazzaroni, S., Corsetti, A., Gobbetti, M. (2000). Purification and Characterization of Novel Antifungal Compounds from the Sourdough Lactobacillus plantarum Strain 21B. Applied and Environmental Microbiology, 66 (9), 4084-4090. doi: https://doi.org/10.1128/aem.66.9.4084-4090.2000

[57] Lavermicocca, P., Valerio, F., Visconti, A. (2003). Antifungal Activity of Phenyllactic Acid against Molds Isolated from Bakery Products. Applied and Environmental Microbiology, 69 (1), 634-640. doi: https://doi.org/10.1128/aem.69.1.634-640.2003

[58] Dal Bello, F., Clarke, C. I., Ryan, L. A. M., Ulmer, H., Schober, T. J., Ström, K. et. al. (2007). Improvement of the quality and shelf life of wheat bread by fermentation with the antifungal strain Lactobacillus plantarum FST 1.7. Journal of Cereal Science, 45 (3), 309-318. doi: https://doi.org/10.1016/j.jcs.2006.09.004

[59] Bover-Cid, S., Holzapfel, W. H. (1999). Improved screening procedure for biogenic amine production by lactic acid bacteria. International Journal of Food Microbiology, 53 (1), 33-41. doi: https://oi.org/10.1016/s0168-1605(99)00152-x

[60] Sunita, S. (2019). Development of starter cultures for fermented functional and /or healthy vegetable and fruit drinks in Project: Development of Functional and Convenience Foods from Agri-horticultural Produce (No CRSCIARI2014023255201 7-18). IRC -I presentation (for official use only).

\title{
APPLICATION OF CO-BIOPROCESSING TECHNIQUES (ENZYMATIC HYDROLYSIS AND FERMANTATION) FOR IMPROVING THE NUTRITIONAL VALUE OF WHEAT BRAN AS FOOD FUNCTIONAL INGREDIENS
}

\author{
Leonid Kaprelyants ${ }^{1}$ \\ leonid@onaft.edu.ua \\ Liliia Pozhitkoval \\ pozhitkova@ukr.net \\ Mykola Buzhylov \\ buzhylovnic@ukr.net \\ ${ }^{1}$ Department of Biochemistry, Microbiology and Physiology of Nutrition \\ Odessa National Academy of Food Technologies \\ 112 Kanatna str., Odessa, Ukraine, 65039
}

\footnotetext{
Abstract

Last time the food industry pays the great attention to questions, connected with changing existing technologies for raising the efficacy of the raw materials complex processing and increasing the output of high-quality products and food ingredients with a minimal amount of waste. Cereal crops are the most reach source of functional ingredients and main component in the human food ration. The technological process of cereal crops processing at enterprises is closely connected with creating a great number of secondary raw material resources and its further utilization.

For confirming the efficacy of using secondary products of grain processing as cheap raw material resources of dietary fiber and physiologically functional ingredients, there is characterized the accessibility of their biotransformation that gives a possibility to get biologically active substances of different chemical nature with a wide spectrum of physiological effects.

Secondary products of cereal crops processing (bran) are multi-component substrates, formed of different histological layers of wheat grains after comminution, consisted of (external pericarp, internal pericarp, grain coat, hyaline and aleurone layer of a grain coat).

Wheat bran is rich in dietary fiber, nutritive and phytochemical substances, that is why, it is most often used for feeding animals. But for today there are important proofs of using it in the food industry.
} 